\title{
CLOUd COMPUTING STRATEGY AND IMPACT IN BANKING/FINANCIAL SERVICES
}

\author{
Prudhvi Parne \\ Information Technology, Bank of Hope, \\ 1655 E Redondo Brach Blvd, Gardena, CA, USA
}

\begin{abstract}
With recent advances in technology, internet has drastically changed the computing world from the concept of parallel computing to distributed computing to grid computing and now to cloud computing. The evolution of cloud computing over the past few years is potentially one of the major advances in the history of computing. Unfortunately, many banks are still hesitant to adopt cloud technology. New technologies such as cloud and AI will have the biggest impacts on the banking industry. For banks and credit unions wanting to achieve greater business agility, cloud technology enables organizations to respond instantly to changing market conditions, leveraging data and applied analytics to achieve customer experience and operational productivity benefits. As a result, cloud computing comes in to provide a solution to such challenges making banking a reliable and trustworthy service. This paper aims at cloud computing strategy, impact in banking and financial institutions and discusses the significant reliance of cloud computing.
\end{abstract}

\section{KEYWORDS}

Cloud Computing, Technology, Finance, Security.

\section{INTRODUCTION}

Banks have always relied on legacy systems from historic times, and in most cases, they are usually reluctant to embrace changes to their technology infrastructure. Nevertheless, with increasing concern for customer data, many banking institutions are now considering shifting to cloud computing. Although the shift is quite intimidating, cloud computing is promising banking institutions to award them immense benefits, which will assist them in overcoming the problems they have been facing with the legacy systems in the recent past. Fintech's recent survey indicated that $46 \%$ of bankers perceive these trials as the most significant obstruction to the development of commercial banks [1]. Therefore, as the banking institutions embark on the journey, the paper will reflect on how the banks will be impacted by the implementation of cloud computing into their daily operations. Cloud computing consists of a set of resources and services offered through the Internet. Hence, cloud computing is also called Internet computing [18].

\section{Cloud Computing}

Cloud computing is defined as a model for enabling banks to have convenient and on-demand network access to a communal pool of configurable computing resources. Such include servers, networks, applications, storage, and services that can be swiftly released and provisioned with little administration effort or the level of interaction with the service provider. Cloud computing promotes accessibility through various attributes, including on-demand services where the client

David C. Wyld et al. (Eds): COMIT, CRBL, BIOM, WiMNeT, SIP, AISO - 2021

pp. 37-45, 2021. CS \& IT - CSCP 2021

DOI: $10.5121 / \mathrm{csit} .2021 .111704$ 
can enjoy unilaterally providing computing abilities [4]. These encompass network storage and server time depending on the organization's requirements without necessarily calling for human interaction with the service provider. Another attribute is broad network access, which is strengthened over the network and can be easily accessed via standard mechanisms that encourage various thick or thin client podiums, including laptops, mobile phones, and PDAs. Resource pooling is the situation where the service provider allows the banking institution to pool their resources such that they can be used to serve numerous clients through the multi-tenant model that is empowered with different virtual and physical resources. These resources can be assigned or reassigned dynamically depending on the consumer demands.

Looking at Cloud Computing Software as a Service (SaaS), we must acknowledge that, this is the package that is used all over the world. In fact, this package allows the provider to license associate use to customers either as a demand or as a service, and this is done through subscription in what is seemingly termed as pay-and-go model. Sometimes this is also increased at no charge in cases where there could be the likelihood of revenue induction from diversified streams apart from the user [6]. For instance, from the promotion or user list sales SaaS which might be growing in the market as already experienced. This anecdote is critical because it is heralding the fact that this software has become a commonplace and so it is critical that various customers and technology users get to know what SaaS connotes and its applications. SaaS offers solutions for organizations and companies which are providing access to various flow of labor, CRM, CMS, analytic and even the third-party services and all these are pegged upon a pay-peruse model [8]. It is also a platform which is professionally administered and protected 24/7 and 365 days a year throughout a remote datacenter. This provides state-of-the-art of Cloud computing technologies [13].

\section{Cloud Computing Models}

Cloud computing promises to improve banking institutions by providing them with a variety of software to select from. The entire cloud architecture [16] is aimed at providing the users with high bandwidth, allowing users to have uninterrupted access to data and applications, on-demand agile network with possibility to move quickly and efficiently between servers or even between clouds and most importantly network security.

The first option is using the software as a service that helps develop banking institutions. Through this software, the micro banking institutions can easily access the internet-hosted software services using the browser instead of relying on traditional applications that have been stored in their server or computer. Depending on software as a service, the application host is responsible for maintaining and controlling the application comprising software settings and updates. This software package has been revered and it has become very popular and fashionable recently for various reasons. For example, it is engaging to the clients because of its shifts in cumbrance and value both the hardware and software package preparation and how it is maintained from the perspective of the client to the seller. SaaS is conjointly offering numerous benefits to the seller. In fact, WHO has recently developed and maintained this application on their platform and have permitted the customer use option of their applications.

The second option is infrastructure as a service which means that the banking institution can buy or rent the disk space and computers from an internet service provider. This will enable them to access information over the internet or private network. The provider maintains the physical computer hardware such as memory, CPU processing, network connectivity, and data storage. Examples of this software include Windows Azure. This is regarded as a fashion for delivering Cloud Computing through an infrastructure-like servers, storage, network associate degrees operative systems and as associate degree-on demand service [7]. It prevents the clients for 
shopping for the servers, datacenters, and software or network instrumentation, rather they can only buy various resources like sometimes an extremely outsourced service on demand.

The last option is the platform as a service that will enable the banking institution to rent operating systems, hardware, network capacity, and storage [17] provided by infrastructure as a service and the corresponding software application and servers environments. Platform as a service offers banks top-notch control over their technical aspects of the computing setup and customize to suit their needs. However, this must be done or applied in the package development environments. PaaS is similarly printed to be a computing platform that allows the establishment of web applications faster and easily and while not the quality of buying and maintenance of the package and infrastructure at a lower end [6]. It is also regarded as analogous to SaaS only that, instead of being package delivered over the web, it is a platform for the creation of the package, delivered over the web.

\section{DifFERENT TyPES OF ClOUd DEPloymentS}

In contrast to the models discussed above, which define how services are offered via the cloud, these different cloud deployment types [11] have to do with where the cloud servers are and who manages them.

The most common cloud deployments are:

Private cloud: A private cloud is a server, data center, or distributed network wholly dedicated to one organization.

Public cloud: A public cloud [12] is a service run by an external vendor that may include servers in one or multiple data centers. Unlike a private cloud, public clouds are shared by multiple organizations. Using virtual machines, individual servers may be shared by different companies, a situation that is called "multitenancy" because multiple tenants are renting server space within the same server.

Hybrid cloud: hybrid cloud deployments combine public and private clouds and may even include on-premises legacy servers. An organization may use their private cloud for some services and their public cloud for others, or they may use the public cloud as backup for their private cloud.

Multi-cloud: multi-cloud is a type of cloud deployment that involves using multiple public clouds. In other words, an organization with a multi-cloud deployment rents virtual servers and services from several external vendors to continue the analogy used above, this is like leasing several adjacent plots of land from different landlords. Multi-cloud deployments can also be hybrid cloud, and vice versa.

\section{Factors Influencing the Adoption of Cloud Computing in BANKS}

From a different perspective, the shift to adopt cloud computing technology in the banking institution is influenced by various factors. The first factor is a classification of information sensitivity in the banking system. Banks have the freedom to select the kind of system suitable for them depending on the data the institution will be processing as permitted by the government regulations and data security [19] necessities. As a result, the banking organization must ensure that the cloud computing they will decide on meets all the qualifications [3]. For instance, the 
United States carefully evaluates their outsourcing procedures and ensure that the banks apply them strictly. The second factor is the competitive advantages and differentiation capacities availed by Cloud Computing. For this, the bank will decide on the choice of computing for the organization depending on the level of competition existing in the industry. As a result, the organization will consider cloud computing an effective alternative to turn to outdo the competitors successfully. Similarly, if there is a need for the bank to differentiate its products, inhouse development is more preferred by the institution owing to its swiftness in marketing its expectations.

\section{Positive Impacts of Cloud Computing on Banks}

Cloud computing has continued to gain popularity as it acts as a catalyst through which banks and financial institutions can rely on transforming the features of their monetary services and tailoring them according to the customers' needs. As banks continue to adopt cloud technology, it becomes essential to propel the institutions towards a positive future. One of Cloud technology's paramount importance to banks and financial institutions is that it creates no need to develop an up-front investment in infrastructure such as software licenses and does not attract the risk of unused licenses. Similarly, cloud technology does not demand investment in hardware components and other associated maintenance services. Therefore, capital expenditure that would have been spent in meeting these qualifications is turned into operational cost, thus enabling the institutions to achieve their goals. The consumers of cloud technology are only needed to use the number of IT resources they initially need and will only be required for the volume of technology they have used.

The second impact of cloud computing on banks and financial institutions includes that cloud computing facilitates fast and easy scaling of the computing resources required to sustain the organization's cloud operations. Cloud technology also plays a crucial role in diminishing ongoing operating, upgrades, and maintenance costs through the utility model. This payback is often described as an instant outcome of the technology. Scaling [15] the technology up and down network capacity, hardware, and cost based on demand can be expensive for large-scale financial institutions because they deal in a wide range of data. Therefore, cloud computing enables banks to easily add or eliminate resources at a fine grain along with a lead time of few minutes instead of several weeks as they wait for the matching of resources to workload closer. Peaks mainly influence this demand for cloud technology resources. The process of waiting can end up attracting a lot of complications for the organization in determining the definite number of servers that an institution requires to execute its tasks without disruption or experiencing data breaches. In most situations, the response regarding the organization's needs is usually based on the cost-benefit analysis. However, with the concept of cloud computing, organizations should forget all about the investigation because the technology offers them a flexible solution for the consistent change in IT resources demand.

Another impact of cloud computing is that it has increased in availability compared to other inhouse solutions by intensifying the accessibility of Virtual Machine, which strengthens the ability of the organization to create a customized environment above the physical infrastructures [2]. Cloud technology enables organizations to access a wide variety of numerous applications and attributes. One of them is Software as a service, a virtualization technique fully exploited when employing the cloud computing model. This is an indication that software applications can be easily accessed via the web interface. The significance is that this application portfolio is getting more dynamic concerning the transformations of the banks as a way of meeting consumer behaviour change. Through cloud computing, applications can be quickly deleted or added to the firm's portfolio within a short period. Nevertheless, cloud computing attracts minimal maintenance costs because it acts as the primary catalyst for innovation. Cloud computing is 
becoming more affordable and more universal, thus creating the opportunity for innovation to continue to grow to new heights.

Besides, cloud computing comes with high data security, thus eliminating the thought of losing valuable data to hackers, which can be drastic to the bank and the customers. In the long run, data breaches tend to be costly, racking up in millions and dollars, thus increasing the opportunity that the institution that has been rigged will be expelled from the market as customers will lose faith in them [5]. while data breach threats are on the rise owing to numerous advancements in technology providing hackers with immense techniques to crack organizations' passwords, cloud computing is paving a long-lasting solution to these problems to hinder hackers from tampering with important information. Cloud computing ensures that the bank has access to an up-to-date customer-centered platform strengthened by complicated password combinations to protect banking data.

Cloud computing has strengthened collaborations where partnership in a cloud environment gives a firm the capability to share and communicate frequently and efficiently outside the old techniques. Cloud computing can provide all workers, sub-contractors and third parties access to all the files, primarily if the banks work in various locations. There is a possibility of choosing a cloud computing model that seems too easy for the banks to share their archives with their advisers. On the contrary, cloud technology is providing banks with improved efficiency in their operations. The financial services provided by these organizations are aided by cloud technology to streamline all the processes using enhanced efficiency. Both sellers and buyers are connected in the payment procedures on a communal application. This is very necessary because it improves the speed of transactions and tracking of data becomes simple. Besides this, cloud computing helps in the continuity of the business since it can assist the financial services firms and banks with fault tolerance, data protection, and recovery from disasters for financial companies. Data Mining is used for extracting potentially useful information from raw data. The implementation of data mining [20] techniques through Cloud computing will allow the users to retrieve meaningful information from virtually integrated data warehouse that reduces the costs of infrastructure and storage.

Moreover, cloud computing incurs lower prices compared with traditional solutions. Nevertheless, agility and transformation are on the list. This involves the financial administrations experiencing shorter development cycles for the novel products via the supple cloud-based operating replicas. The associated technology ropes the quicker and more efficient replies to the requirements of the latest banking customers [5]. It helps the firm shift non-critical facilities, including software patches, maintenance, and other computing matters. This aids the financial companies to concentrate more on business growth and expansion. Also, the flexibility of the work practices allows the employees to be flexible, especially in their work practices. For instance, increase the capacity to access data from homesteads, on public holidays, or even through the commute to and from the job. If there is a need to access the data while off-site, it is possible to connect to the virtual office faster and straightforwardly. Finally, is the access to automatic updates. The necessities can be involved in the service fee. The system will often be updated with modern technology depending on the cloud computing service provided. The current date versions of software can be included and the advancements to servers and computer dispensation power.

\section{Negative ImPaCts OF Cloud COMPUTING}

Despite the various aids of cloud computing knowledge, most financial institutions are still not able to adopt it. Some of the challenges that are barriers to the banks from implementing it are data and security privacy. Sensitive information is contained in the bank data and keeping it safe 
from a cyber-breach is a must for all banking sectors. There is no exception regardless of the technology; security has to be tight and remains an issue. The security breaches occurrences are inventible but avoidable [2]. Also, regulatory and compliance are considered where all banks are authorized to comply with strict standards. A lot of the banking regulators need the client's financial information situated in the same nation. Specific compliance guidelines need treasured data not to be mixed with the other data on the database or shared servers. The Data Cloud [14] allows organizations to unify and connect to a single copy of all of their data with ease. The result is an ecosystem of thousands of businesses and organizations connecting to not only their own data, but also connecting to each other by effortlessly sharing and consuming shared data and data services. Lastly, there is no complete control of severe firms' submissions, and data is an essential concern for financial organizations. If a third-party handles cloud service providers, they might lessen the ability to be supple and elegant. Therefore, not having control of an enormous volume of data dissuades organizations from moving to the cloud.

\section{Case Study}

Deutsch Bank is a financial institution offering investment and commercial banking, transaction banking, retail banking along with wealth administration services and products to organizations, organizational financiers and government, private individuals and large and small businesses. Deutsch Bank is among the Germany's well performing banking institutions with a robust brand position across Europe as well as robust presence in Asia Pacific and American regions. Google on the other hand is a platform that offers organizations with leading infrastructure, industrial solutions and also enhances platform capabilities. The platform's objective is to deliver top-notch cloud solutions that leverages Google's superior technology to assist corporations to function more effectively and adjust to the transforming wants of their consumers as a way of providing them with a firm foundation for their future financing decisions. customers across 150 countries have been turning to Google cloud as their reliable partner to address the dire business challenges facing them.

Google Cloud and Deutsch bank entered a multi-year contract in July and with the ink already dried up now, these organizations have proceeded to outline a number of ways that the bank will be using the digitalization of their processes. The 150-year-old banking institution is looking forward to utilizing the Google cloud for several number of their upcoming products. These comprises of innovative loaning products to offer upkeep to 'pay per use' systems as a way of providing alternative to procuring assets outright and developing a freshly innovated interface for consumption by their clients. The company's Autobahn podium which is tasked with providing access to Deutsch Bank research, analytics and commentary will also be getting a makeover to establish more individualized experiences.

On a more typical theme, providing robust security is a slogan for Deutsch Bank as it will provide them with high level functionality and administer their encryption keys and select the data analytics region that can be used in deploying the applications. With the improvement, resilience and flexibility is expected to improve significantly without compromising the organization's goal on providing privacy and security to secure customer information as well as the Deutsch Bank information assets. This collaboration will be a significant win for both Google cloud and Deutsch Bank releasing proposal in February. The utilization of shift to the cloud to the press materials proposes a significant overhaul. Regarding to the new statement released in October of 2019, an internal memo highlighted a total of $\$ 12$ billion investments for the technology with an assurance to strengthen cloud computing strategy. For Google, Deutsch Bank was a perfect match into one of the three chief consumer verticals of healthcare, finance and retail. 
According to Deutsche Bank's chief technology, innovation and data officer, Leukert, the new chapter to the bank as it would open numerous opportunities to the institutions in the long run. The officer continued that with Google Cloud by their side, they will be able to have a strategic partner that will play a significant role in accelerating their technological transformation enabling them to utilize a safe and flexible environment for them to swiftly deliver new services and products to their users [9]. The officer continued that this transformation to join the cloud computing is a blueprint to bring together the respective strengths inside technology and banking for the benefits of the Deutsche clients.

Deutsch Bank and Google Cloud are developing a plan that will precisely co-innovate with numerous encouraging establishments to Fintech by providing accessibility to a wide implementation of the bank's cloud-native offerings. The bank's client will benefit significantly from the innovation as it will play a great role in reshaping how the bank will be designing and delivering their financial products and services. With rapid application development, along with the effective usage of forward-thinking artificial intelligent and wide-ranging data analysis gears, the organization will be in a profitable position to respond to the increasing flexibility and accurately solve pressing problems, customer needs and trends.

Additionally, Robin Enslin the president of Google cloud pointed that the cloud computing will also focus on advancing the company's Mobile self-service selections, artificial intelligenceoriented endorsements and other helpful novelties, the company expects that the customer's banking experience will transform greatly [10]. The president continues that the partnership will play a great role in reviving numerous innovations and further develop the bank to emerge as the best early expertise adopter. Deutsch Bank is a pioneer in the service industry and the Google Cloud computing pointed out that they could not be more ecstatic to partner with such an important market leader.

These partners are also considering various areas to explore to make the best out of their partnerships. Such include providing new lending commodities to provide ample support to the pay-per-use systems as a brilliant alternative to secure the right asset outright. The partners are also developing an intuitive and unified interface for the retail consumers across their member countries to more easily access the various products offered by Postbank and Deutsche bank products. Lastly, Google Cloud and Deutsch banks are exploring numerous strategies to aid in enhancing the Autobahn banking platform to become an award-winning electronic service that will be providing organizational and company clients with the power to establish more individualized experiences and recommendations. In attempt to expand their customer outreach, Deutsch bank is making strategies to list all Google Cloud merchandises on the Google Cloud marketplace to propel a wider implementation of the institution's new cloud native solutions and services. Significantly, the shift to the cloud computing will provide the Deutsch Bank with an upper hand of exploiting an up-to-date information and fully administered atmosphere for their applications. With the new partnership underway, Deutsch Bank will be able to select the type of datacenter region that will work well with their applications while they are being deployed to accommodate for data location roles or preferences. The bank's applications will be in a position to encrypt data both in transit and at rest. Google Cloud will also offer the Deutsche bank with the functionality it needs to allow the bank to effectively manage their chief encryption keys.

\section{Conclusions}

As a concluding remark, it is clear that cloud computing has completely transformed the face of banking. Cloud computing have brought more affordable and reliable means through which banks can rely on to ensure that their customers information is well protected. Other positive impacts include its high scalability levels and vast capacities to support collaboration between 
different shareholders in the organization by allowing free exchange of information among them. Despite the positivity, cloud computing has also brought along some among them including that the costs are still extremely high for micro enterprises to adopt it.

\section{REFERENCES}

[1] Tiwari, S., Bharadwaj, S., and Joshi, S. (2021). A Study of Impact of Cloud Computing and Artificial Intelligence on Banking Services, Profitability and Operational Benefits. Turkish Journal of Computer and Mathematics Education (TURCOMAT), 12(6), 1617-1627.

[2] Kaya, F., Van Den Berg, M., Wieringa, R., and Makkes, M. (2020, June). The Banking Industry Underestimates Costs of Cloud Migrations. In 2020 IEEE 22nd Conference on Business Informatics (CBI) (Vol. 1, pp. 300-309). IEEE.

[3] Rieger, P., Gewald, H., and Schumacher, B. (2013). Cloud-computing in banking influential factors, benefits and risks from a decision maker's perspective.

[4] Parry, R., and Bisson, R. (2020). Legal approaches to management of the risk of cloud computing insolvencies. Journal of Corporate Law Studies, 20(2), 421-451.

[5] Kshetri, N. (2010, January). Cloud computing in developing economies: drivers, effects, and policy measures. In Proceedings of PTC (pp. 1-22).

[6] Marcel Decker, "Security of the Internet", The Froehlich/kent reference work of Telecommunications vol, 15.

[7] www.doc.ic.ac.uk/ nd/surprise_96/journal/vol4/vk5/ report.html.

[8] Rerns Grobauer Tobias Walloschek, Elmar Stocker,Co published by IEEE Computer and Reliability Societies" March-2011, Pg 53.

[9] Ali, A. (2021). Case Study-Deutsche Bank.

[10] Villar, A. S., \& Khan, N. (2021). Robotic process automation in banking industry: a case study on Deutsche Bank. Journal of Banking and Financial Technology, 1-16.

[11] Ali, A. (2021). Case Study-Deutsche Bank.

[10] Villar, A. S., \& Khan, N. (2021). Robotic process automation in banking industry: a case study on Deutsche Bank. Journal of Banking and Financial Technology, 1-16.

[11] S Sokolov, O Idiriz, M Vukadinoff, S Vlaev (2020). Scaling and automation in cloud deployments of enterprise applications, Journal of Engineering Science and Technology Review jestr.org.

[12] B Hayes (2008). Cloud computing, https://dl.acm.org/doi/fullHtml/10.1145/1364782.1364786 dl.acm.org.

[13] L Wang, G Von Laszewski, A Younge, X He, M Kunze, J Tao \& C Fu (2010). Cloud computing: a perspective study generation computing, 2010. Springer.

[14] N Antonopoulos, L Gillam (2017). Cloud computing. Springer, 191-233.

[15] M Armbrust, A Fox, R Griffith, AD Joseph, R Katz, A Konwinski, G Lee, D Patterson, A Rabkin, I Stoica, M Zaharia (2010). A view of cloud computing, dl.acm.org. Volume 53, Number 4 (2010), Pages 50-58.

[16] Y Jadeja, K Modi (2012). Cloud computing-concepts, architecture and challenges. International Conference on Computing, Electronics and Electrical Technologies (ICCEET). IEEE.

[17] DC Marinescu - 2017. Cloud computing: theory and practice, second edition. 195-230.

[18] MNO Sadiku, SM Musa, OD Momoh (2014). Cloud computing: opportunities and challenges. IEEE potentials, Volume: 33, Issue: 1, Jan.-Feb. 2014. IEEE.

[19] JF Ransome (2017). Cloud Computing: Implementation, Management, and Security. taylorfrancis.com. 153-182.

[20] M Rambabu, S Gupta, RS Singh (2021). Data Mining in Cloud Computing: Survey. Innovations in Computational Intelligence and Computer Vision. Springer, pp 48-56. 


\section{AUTHORS}

Prudhvi Parne received the Master's (MS) degree in Computer Science from University of Louisiana, Lafayette, LA, USA. His expertise spans in the areas of Cloud Architecture, Software Development, Finance, Banking, Hybrid clouds, Product Management, and Product leadership.

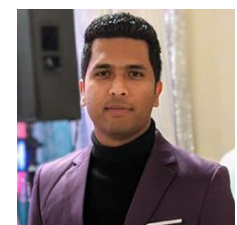

(C) 2021 By AIRCC Publishing Corporation. This article is published under the Creative Commons Attribution (CC BY) license. 